\title{
Complete genome sequence of Leptotrichia buccalis type strain $\left(C-1013-b^{\top}\right)$
}

\author{
Natalia Ivanova ${ }^{1}$, Sabine Gronow ${ }^{2}$, Alla Lapidus ${ }^{1}$, Alex Copeland ${ }^{1}$, Tijana Glavina Del Rio ${ }^{1}$, \\ Matt Nolan ${ }^{1}$, Susan Lucas ${ }^{1}$, Feng Chen ${ }^{1}$, Hope Tice ${ }^{1}$, Jan-Fang Cheng ${ }^{1}$, Elizabeth Saunders ${ }^{1,3}$, \\ David Bruce $^{1,3}$, Lynne Goodwin ${ }^{1,3}$, Thomas Brettin ${ }^{1,3}$, John C. Detter ${ }^{1,3}$, Cliff Han ${ }^{1,3}$, Sam Pit- \\ luck ${ }^{1}$, Natalia Mikhailova ${ }^{1}$, Amrita Pati ${ }^{1}$, Konstantinos Mavrommatis ${ }^{1}$, Amy Chen ${ }^{4}$, Krishna \\ Palaniappan $^{4}$, Miriam Land ${ }^{1,5}$, Loren Hauser ${ }^{1,5}$, Yun-Juan Chang ${ }^{1,5}$, Cynthia D. Jeffries ${ }^{1,5}$, Pa- \\ trick Chain ${ }^{1,6}$, Christine Rohde ${ }^{2}$, Markus Göker ${ }^{2}$, Jim Bristow ${ }^{1}$, Jonathan A. Eisen ${ }^{1,7}$, Victor \\ Markowitz ${ }^{4}$, Philip Hugenholtz ${ }^{1}$, Nikos C. Kyrpides ${ }^{1}$, and Hans-Peter Klenk ${ }^{2 *}$ \\ 1 DOE Joint Genome Institute, Walnut Creek, California, USA \\ 2 DSMZ - German Collection of Microorganisms and Cell Cultures $\mathrm{GmbH}$, Braunschweig, \\ Germany \\ 3 Los Alamos National Laboratory, Bioscience Division, Los Alamos, New Mexico USA \\ 4 Biological Data Management and Technology Center, Lawrence Berkeley National Labor- \\ atory, Berkeley, California, USA \\ 5 Oak Ridge National Laboratory, Oak Ridge, Tennessee, USA \\ 6 Lawrence Livermore National Laboratory, Livermore, California, USA \\ 7 University of California Davis Genome Center, Davis, California, USA
}

*Corresponding author: Hans-Peter Klenk

Keywords: Fusobacteria, 'Leptotrichiaceae', Gram-negative fusiform rods, human oral microflora, dental plaque, non-motile, non-sporulating, anaerobic

Leptotrichia buccalis (Robin 1853) Trevisan 1879 is the type species of the genus, and is of phylogenetic interest because of its isolated location in the sparsely populated and neither taxonomically nor genomically adequately accessed family 'Leptotrichiaceae' within the phylum 'Fusobacteria'. Species of Leptotrichia are large, fusiform, non-motile, non-sporulating rods, which often populate the human oral flora. L. buccalis is anaerobic to aerotolerant, and saccharolytic. Here we describe the features of this organism, together with the complete genome sequence and annotation. This is the first complete genome sequence of the order 'Fusobacteriales' and no more than the second sequence from the phylum 'Fusobacteria'. The 2,465,610 bp long single replicon genome with its 2306 protein-coding and 61 RNA genes is a part of the Genomic Encyclopedia of Bacteria and Archaea project.

\section{Introduction}

Strain C-1013-bT (= DSM 1135 = ATCC $14201=$ JCM 12969) is the type strain of Leptotrichia buccalis [1], which is the type species of the genus first adequately described in 1879 by Trevisan to accommodate the oral filamentous bacteria and to separate them from the algae [2,3]. For a while, two entirely different organisms were termed $L$. buccalis in the literature [3]. One of these was 'Leptothrix buccalis', a name originally employed by Robin in 1853 for filamentous forms which he had seen in wet mounts of tooth scrapings [4]. Over a century of the history of classification and misclassification of L. buccalis was documented by Gilmore et al. 1961 [3]. L. buccalis was among the first bacteria to be described and drawn in the letters of Antoni van Leeuwenhoek [5]. Next to Fusobacterium nucleatum [6], L. buccalis is only the second species from the phylum Fusobacteria for which a complete genome sequence is described. Here we present a summary classification and a set of features for $L$. buccalis strain C-1013- $\mathrm{b}^{\mathrm{T}}$ together with the description of the complete genomic sequencing and annotation. 


\section{Classification and features}

The primary habitat of $L$. buccalis and most other Leptotrichia species is the human oral cavity, especially dental plaque. L. buccalis also is found in the female genitourinary tract and the intestinal tract [11,13]. Although L. buccalis and L. buccalis-like bacteria have also occasionally been recovered from blood, mostly in immunocompromised patients, they are not known as causative agents of systemic infections $[11,14]$ even though an endotoxin was documented for the L. buccalis $[5,14]$. Almost all of the cultivated Leptotrichia isolates cluster in 16S rRNA sequence comparisons with one of the five other type strains of the genus Leptotrichia [11] (Figure 1). Except for the uncultured clone GI5-008-C04 (FJ192568), which has been recovered from screen- ing of a spacecraft assembly clean room during the Phoenix mission, all significantly related phylotypes were from the usual habitats as described above. No phylotypes from environmental screening or genomic surveys could be linked with more than $85 \% 16 \mathrm{~S}$ rRNA sequence similarity to L. buccalis (status May 2009).

Figure. 1 shows the phylogenetic neighborhood of $L$. buccalis strain C-1013- $\mathrm{b}^{\mathrm{T}}$ in a $16 \mathrm{~S}$ rRNA based tree. The sequences of the five 16S rRNA gene copies in the genome of strain C-1013-b ${ }^{\mathrm{T}}$ differ from each other by 5 to 20 nucleotides (up to $1.3 \%$ ), and by 4 to 16 nucleotides plus 38 ambiguities (total up to $3.6 \%$ ) from the previously published 16S rRNA sequence generated from NCTC 10429 (X90831)

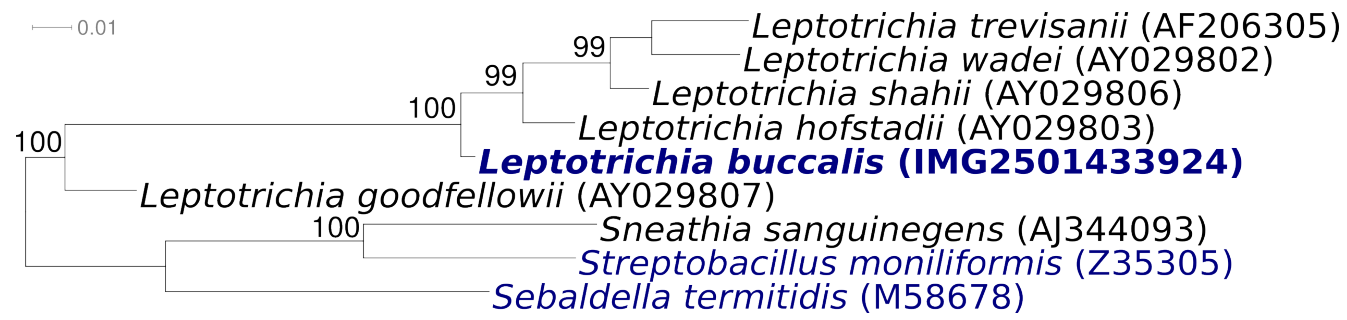

Figure 1. Phylogenetic tree highlighting the position of $L$. buccalis $C-1013-b^{\top}$ relative to all type strains of the genus Leptotrichia inferred from 1,421 aligned characters [15,16] of the 16S rRNA sequence under the maximum likelihood criterion [17], and rooted with all type strains of the family Leptotrichiaceae. The branches are scaled in terms of the expected number of substitutions per site. Numbers above branches are support values from 1,000 bootstrap replicates, if larger than 60\%. Lineages with type strain genome sequencing projects registered in GOLD [18] are shown in blue, published genomes in bold.

Older cells of L. buccalis strain C-1013-b ${ }^{\mathrm{T}}$ are Gram negative, but younger cells that have been in culture for less than six hours are Gram-positive Table 1 [5]. The organism forms long rods, commonly occurring in pairs, and is non-motile [5] (Figure 2). Young colonies are colorless, smooth, shiny, raised and described as "medusa-head" colonies because of filamentous edges [5]. On first isolation, L. buccalis is anaerobic but becomes aerotolerant upon transfer and grows in the presence of air and $\mathrm{CO}_{2}$ [5,13]. L. buccalis is susceptible to many antibiotics but resistant to aminoglycosides [5]. The organism is highly saccharolytic and ferments a range of different sugars $[5,13]$. The main metabolic end product is lactic acid [13]. The $\mathrm{G}+\mathrm{C}$ content was already described in 1982 as 'unusually low' (25\%) [5].

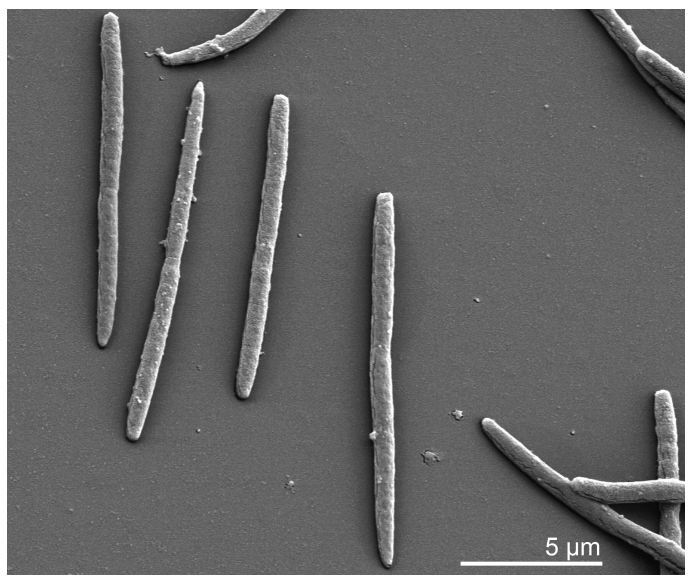

Figure 2. Scanning electron micrograph of L. buccalis stain C-1013-b ${ }^{\top}$ (Manfred Rohde, Helmholz Centre for Infection Research, Braunschweig). 
Table 1. Classification and general features of L. buccalis strain C-1013- $\mathrm{b}^{\mathrm{T}}$ according to the MIGS recommendations [7]

\begin{tabular}{|c|c|c|c|}
\hline MIGS ID & Property & Term & $\begin{array}{l}\text { Evidence } \\
\text { code }\end{array}$ \\
\hline & \multirow{8}{*}{ Current classification } & Domain Bacteria & TAS [8] \\
\hline & & Phylum 'Fusobacteria' & TAS [9] \\
\hline & & Class 'Fusobacteria' & TAS [9] \\
\hline & & Order 'Fusobacteriales' & TAS [9] \\
\hline & & Family 'Leptotrichiaceae' & \\
\hline & & Genus Leptotrichia & TAS [2] \\
\hline & & Species Leptotrichia buccalis & TAS [2] \\
\hline & & Type strain C-1013-b & TAS [1] \\
\hline & Gram stain & negative & TAS [5] \\
\hline & Cell shape & long rods & TAS [5] \\
\hline & Motility & nonmotile & TAS [5] \\
\hline & Sporulation & nonsporulating & TAS [5] \\
\hline & Temperature range & mesophile & NAS \\
\hline & Optimum temperature & $37^{\circ} \mathrm{C}$ & NAS \\
\hline & Salinity & normal & NAS \\
\hline \multirow[t]{3}{*}{ MIGS-22 } & Oxygen requirement & $\begin{array}{l}\text { anaerobic on isolation, becomes aerotole- } \\
\text { rant on further transfer }\end{array}$ & TAS [5] \\
\hline & Carbon source & mono- and disaccharides & TAS [5] \\
\hline & Energy source & carbohydrates & NAS \\
\hline MIGS-6 & Habitat & oral cavities & TAS [5] \\
\hline MIGS-15 & Biotic relationship & free living & NAS \\
\hline \multirow[t]{3}{*}{ MIGS-14 } & Pathogenicity & opportunistic pathogen & TAS [5] \\
\hline & Biosafety level & 1 & TAS [10] \\
\hline & Isolation & human oral flora & TAS [11] \\
\hline MIGS-4 & Geographic location & global & NAS \\
\hline MIGS-5 & Sample collection time & mid of $19^{\text {th }}$ century & TAS [3] \\
\hline MIGS-4.1 & Latitude - Longitude & not reported & \\
\hline MIGS-4.2 & & & \\
\hline MIGS-4.3 & Depth & not reported & \\
\hline MIGS-4.4 & Altitude & not reported & \\
\hline
\end{tabular}

Evidence codes - IDA: Inferred from Direct Assay (first time in publication); TAS: Traceable Author Statement (i.e., a direct report exists in the literature); NAS: Non-traceable Author Statement (i.e., not directly observed for the living, isolated sample, but based on a generally accepted property for the species, or anecdotal evidence). These evidence codes are from the Gene Ontology project [12]. If the evidence code is IDA, then the property was observed for a living isolate by one of the authors or an expert mentioned in the acknowledgements.

\section{Genome sequencing}

\section{Genome project history}

This organism was selected for sequencing on the basis of its phylogenetic position, and is part of the Genomic Encyclopedia of Bacteria and Archaea project. The genome project is deposited in the Genomes OnLine Database [18] and the complete genome sequence is deposited in GenBank. Sequencing, finishing and annotation were performed by the
DOE Joint Genome Institute (JGI). A summary of the project information is shown in Table 2

\section{Growth conditions and DNA isolation}

L. buccalis strain C-1013-b ${ }^{\mathrm{T}}$ (DSM 1135) was grown anaerobically in DSMZ medium 104 (modified PYGMedium) at $37^{\circ} \mathrm{C}$. DNA was isolated from 1-1.5 $\mathrm{g}$ of cell paste using Qiagen Genomic 500 DNA Kit (Qiagen, Hilden, Germany) following the manufacturer's instructions. 
Table 2. Genome sequencing project information

\begin{tabular}{|c|c|c|}
\hline MIGS ID & Property & Term \\
\hline MIGS-31 & Finishing quality & Finished \\
\hline MIGS-28 & Libraries used & $\begin{array}{l}\text { Three genomic libraries: two Sanger } \\
\text { libraries - } 8 \text { kb pMCL200 and fosmid } \\
\text { pcc1Fos and one } 454 \text { pyrosequence } \\
\text { standard library }\end{array}$ \\
\hline MIGS-29 & Sequencing platforms & ABI3730, 454 GS FLX \\
\hline MIGS-31.2 & Sequencing coverage & $9.7 \times$ Sanger; $42 \times$ pyrosequence \\
\hline MIGS-30 & Assemblers & Newbler version 1.1.02.15, phrap \\
\hline \multirow[t]{6}{*}{ MIGS-32 } & Gene calling method & Prodigal \\
\hline & INSDC / Genbank ID & CP001685 \\
\hline & Genbank Date of Release & September 1, 2009 \\
\hline & GOLD ID & Gc01090 \\
\hline & NCBI project ID & 29445 \\
\hline & Database: IMG-GEBA & 2501416906 \\
\hline \multirow[t]{2}{*}{ MIGS-13 } & Source material identifier & DSM 1135 \\
\hline & Project relevance & Tree of Life, GEBA \\
\hline
\end{tabular}

\section{Genome sequencing and assembly}

The genome was sequenced using a combination of Sanger and 454 sequencing platforms. All general aspects of library construction and sequencing performed at the JGI can be found at http://www.jgi.doe.gov/. 454 Pyrosequencing reads were assembled using the Newbler assembler version 1.1.02.15 (Roche). Large Newbler contigs were broken into 2,747 overlapping fragments of 1,000 bp and entered into assembly as pseudo-reads. The sequences were assigned quality scores based on Newbler consensus q-scores with modifications to account for overlap redundancy and to adjust inflated q-scores. A hybrid 454/Sanger assembly was made using the parallel phrap assembler (High Performance Software, LLC). Possible mis-assemblies were corrected with Dupfinisher or transposon bombing of bridging clones [21]. Gaps between contigs were closed by editing in Consed, custom primer walk or PCR amplification. A total of 908 Sanger finishing reads were produced to close gaps, to resolve repetitive regions, and to raise the quality of the finished sequence. The error rate of the completed genome sequence is less than 1 in 100,000. Together all sequence types provided $51.7 \times$ coverage of the genome. The final assembly contains 28,754 Sanger reads in addition to the 454 based pseudo reads.

\section{Genome annotation}

Genes were identified using Prodigal [22] as part of the Oak Ridge National Laboratory genome annotation pipeline, followed by a round of manual curation using the JGI GenePRIMP pipeline (http://geneprimp.jgi-psf.org) [23]. The predicted CDSs were translated and used to search the National Center for Biotechnology Information (NCBI) nonredundant database, UniProt, TIGRFam, Pfam, PRIAM, KEGG, COG, and InterPro databases. Additional gene prediction analysis and functional annotation was performed within the Integrated Microbial Genomes (IMG-ER) platform [24].

\section{Genome properties}

The genome is 2,345,610 bp long and comprises one circular chromosome with a $29.7 \%$ GC content (Table 3 and Figure 3). Of the 2367 genes predicted, 2306 were protein coding genes, and 61 RNAs; 86 pseudogenes were also identified. The majority of the genes $(65.4 \%)$ were assigned with a putative function while those remaining were annotated as hypothetical proteins. The properties and the statistics of the genome are summarized in Table 3. The distribution of genes into COGs functional categories is presented in Table 4. 


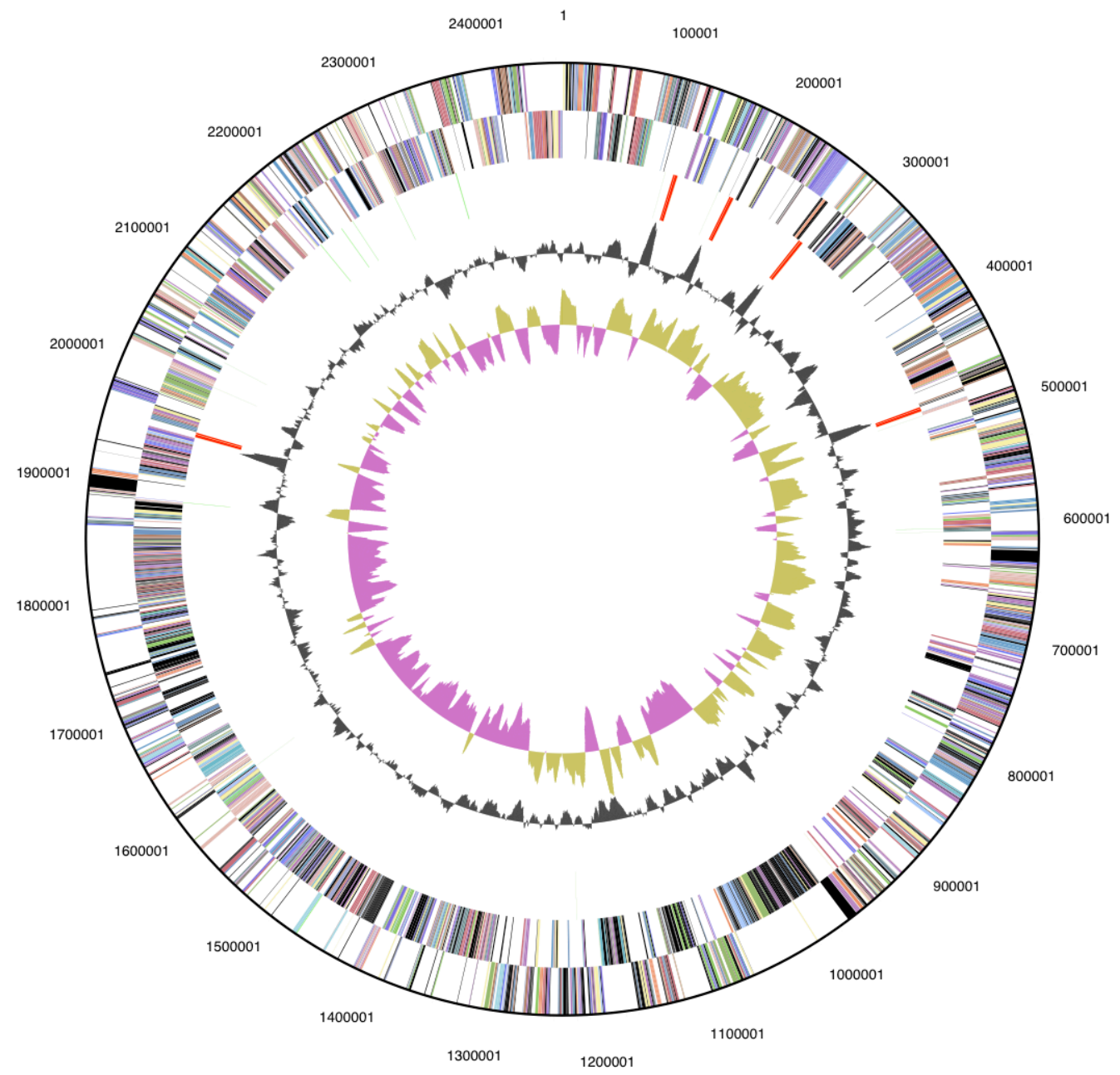

Figure 3. Graphical circular map of the genome. From outside to the center: Genes on forward strand (color by COG categories), Genes on reverse strand (color by COG categories), RNA genes (tRNAs green, rRNAs red, other RNAs black), GC content, GC skew.

Table 3. Genome Statistics

\begin{tabular}{lrr}
\hline Attribute & \multicolumn{1}{c}{ Value } & \% of Total \\
\hline Genome size (bp) & $2,465,610$ & $100.00 \%$ \\
DNA Coding region (bp) & $2,139,206$ & $86.76 \%$ \\
DNA G+C content (bp) & 730,947 & $29.65 \%$ \\
Number of replicons & 1 & \\
Extrachromosomal elements & 0 & \\
Total genes & 2,367 & $100.00 \%$ \\
RNA genes & 61 & $2.58 \%$ \\
rRNA operons & 5 & \\
Protein-coding genes & 2,306 & $97.42 \%$ \\
Pseudo genes & 86 & $3.63 \%$ \\
Genes with function prediction & 1,547 & $65.36 \%$ \\
Genes in paralog clusters & 402 & $16.98 \%$ \\
Genes assigned to COGs & 1,533 & $64.77 \%$ \\
Genes assigned Pfam domains & 1,577 & $66.62 \%$ \\
Genes with signal peptides & 432 & $18.25 \%$ \\
Genes with transmembrane helices & 530 & $22.39 \%$ \\
CRISPR repeats & 4 & \\
\hline
\end{tabular}


Table 4. Number of genes associated with the general COG functional categories

\begin{tabular}{lrrl}
\hline Code & Value & \% age & Description \\
\hline $\mathrm{J}$ & 144 & 6.2 & Translation, ribosomal structure and biogenesis \\
$\mathrm{A}$ & 0 & 0.0 & RNA processing and modification \\
$\mathrm{K}$ & 86 & 3.7 & Transcription \\
$\mathrm{L}$ & 124 & 5.4 & Replication, recombination and repair \\
$\mathrm{B}$ & 0 & 0.0 & Chromatin structure and dynamics \\
$\mathrm{D}$ & 24 & 1.0 & Cell cycle control, mitosis and meiosis \\
$\mathrm{Y}$ & 0 & 0.0 & Nuclear structure \\
$\mathrm{V}$ & 28 & 1.2 & Defense mechanisms \\
$\mathrm{T}$ & 47 & 2.0 & Signal transduction mechanisms \\
$\mathrm{M}$ & 112 & 4.9 & Cell wall/membrane biogenesis \\
$\mathrm{N}$ & 6 & 0.3 & Cell motility \\
$\mathrm{Z}$ & 0 & 0.0 & Cytoskeleton \\
$\mathrm{W}$ & 0 & 0.0 & Extracellular structures \\
$\mathrm{U}$ & 34 & 1.5 & Intracellular trafficking and secretion \\
$\mathrm{O}$ & 68 & 3.0 & Posttranslational modification, protein turnover, chaperones \\
$\mathrm{C}$ & 79 & 3.4 & Energy production and conversion \\
$\mathrm{G}$ & 110 & 4.8 & Carbohydrate transport and metabolism \\
$\mathrm{E}$ & 176 & 7.6 & Amino acid transport and metabolism \\
$\mathrm{F}$ & 54 & 2.3 & Nucleotide transport and metabolism \\
$\mathrm{H}$ & 80 & 3.5 & Coenzyme transport and metabolism \\
$\mathrm{I}$ & 44 & 1.9 & Lipid transport and metabolism \\
$\mathrm{P}$ & 84 & 3.6 & Inorganic ion transport and metabolism \\
$\mathrm{Q}$ & 11 & 0.5 & Secondary metabolite biosynthesis, transport and catabolism \\
$\mathrm{R}$ & 206 & 8.9 & General function prediction only \\
$\mathrm{S}$ & 141 & 6.1 & Function unknown \\
- & 773 & 28.1 & Not in COGs \\
\hline
\end{tabular}

\section{Acknowledgements}

We would like to gratefully acknowledge the help of Sabine Welnitz for growing L. buccalis cultures and Susanne Schneider for DNA extraction and quality analysis (both at DSMZ). This work was performed under the auspices of the US Department of Energy Office of Science, Biological and Environmental Research Program, and by the University of California, Law-

\section{References}

1. Skerman VBD, McGowan V, Sneath PHA. Approved list of bacterial names. Int I Syst Bacteriol 1980; 30: 225-230.

2. Trevisan V. Prime linee d'introduzione allo studio dej Batterj italiani. Rend Reale Inst Lombardo Sci Ser II 1879; 12: 133-151.

3. Gilmour MN, Howell A, Jr., Bibby BG. The classification of organisms termed Leptotrichia (Leptothrix) buccalis. Bacteriol Rev 1961; 25: 131141. PubMed rence Berkeley National Laboratory under contract No. DE-AC02-05CH11231, Lawrence Livermore National Laboratory under Contract No. DE-AC5207NA27344, and Los Alamos National Laboratory under contract No. DE-AC02-06NA25396, as well as German Research Foundation (DFG) INST 599/1-1

4. Robin C. Histoire naturelle des evégétaux parasites qui croissent sur l'homme et sur les animaux vivantis, JB Baillière, Paris 1853; pp 345-454.

5. Hofstad T. The Genus Leptotrichia. In: The Prokaryotes Vol. IV, 2nd ed. 1992, Springer-Verlag New York.

6. Kapatral V, Anderson I, Ivanova N, Reznik G, Los T, Lykidis A, Bhattacharyya A, Bartman A, Gardner W, Grechkin G, et al. Genome sequence and analysis of the oral bacterium Fusobacterium nuc- 
leatum strain ATCC 25586. J Bacteriol 2002; 184: 2005-2018. PubMed doi:10.1128/JB.184.7.2005$\underline{2018.2002}$

7. Field D, Garrity G, Gray T, Morrison N, Selengut J, Sterk P, Tatusova T, Thomson N, Allen MJ, Angiuoli SV, et al. Towards a richer description of our complete collection of genomes and metagenomes: the "Minimum Information about a Genome Sequence" (MIGS) specification. Nat Biotechnol 2008; 26: 541-547. PubMed doi: $10.1038 / \mathrm{nbt} 1360$

8. Woese CR, Stackebrandt E, Macke TJ. Fox GE. A phylogenetic definition of the major eubacterial taxa. Syst Appl Microbiol 1985; 6: 143-151. PubMed

9. Garrity GM, Holt J. In: Garrity GM, Boone DR, Castenholz RW (2001). Taxonomic Outline of the Archaea and Bacteria. Bergey's Manual of Systematic Bacteriology, 2nd Ed. Volume 1 The Archaea, Deeply Branching and Phototrophic Bacteria. Springer-Verlag, New York pp 155-166

10. Anonymous. Biological Agents. Technical rules for biological agents www.baua.de

11. Eribe ERK, Paster BJ, Caugant DA, Dewhirst FE, Stromberg VK, Lykidis A, Bhattacharyya A, Bartman A, Gardner W, Grechkin G, et al. Genetic diversity of Leptotrichia and description of Leptotrichia goodfellowii sp. nov., Leptotrichia hofstadii sp. nov., Leptotrichia shahii sp. nov. and Leptotrichia wadei sp. nov. Int I Syst Evol Microbiol 2004; 54: 583-592. PubMed PubMed doi:10.1099/ijs.0.02819-0

12. Ashburner M, Ball CA, Blake JA, Botstein D, Butler $\mathrm{H}$, Cherry JM, Davis AP, Dolinski K, Dwight SS, Eppig JT, et al. The Gene Ontology Consortium. Gene ontology: tool for the unification of biology. Nat Genet 2000; 25: 25-29. PubMed doi:10.1038/75556

13. Hofstad T, Jantzen E. Fatty Acids of Leptotrichia buccalis: Taxonomic Implications. J Gen Microbiol 1982; 128: 151-153.

14. Bhally HS, Lema C, Borek A, Carroll KC. Leptotrichia buccalis bacteremia in two patients with acute myelogenous leukemia. Infectious Diseases J of Pakistan 2004; Apr-Jun:49-51.

15. Lee C, Grasso C, Sharlow MF. Multiple sequence alignment using partial order graphs. Bioinformat- ics 2002; 18: 452-464. PubMed

doi:10.1093/bioinformatics/18.3.452

16. Castresana J. Selection of conserved blocks from multiple alignments for their use in phylogenetic analysis. Mol Biol Evol 2000; 17: 540-552. PubMed PubMed

17. Stamatakis A, Hoover P, Rougemont J. A rapid bootstrap algorithm for the RAxML web-servers. Syst Biol 2008; 57: 758-771. PubMed doi:10.1080/10635150802429642

18. Liolios K, Mavromatis K, Tavernarakis N, Kyrpides NC. The Genomes OnLine Database (GOLD) in 2007: status of genomic and metagenomic projects and their associated metadata. Nucleic Acids Res 2007; 36: D475-D479. PubMed doi:10.1093/nar/gkm884

19. Fukuhara $\mathrm{H}$, Umemoto $\mathrm{T}$, Sagawa $\mathrm{H}$, Kato K, Kotani S. Purification and quantitative chemical analysis of cell wall peptidoglycans of Leptotrichia buccalis. Infect Immun 1983; 39: 132-136. PubMed

20. Schleifer KH, Kandler O. Peptidoglycan types of bacterial cell walls and their taxonomic implications. Bacteriol Rev 1972; 36: 407-477. PubMed PubMed

21. Sims D, Brettin T, Detter JC, Han C, Lapidus A, Copeland A, Glavina Del Rio T, Nolan M, Chen F, Lucas S, et al. Complete genome of Kytococcus sedentarius type strain (strain 541T). Stand Genomic Sci 2009; 1: 12-20. doi:10.4056/sigs.761

22. Anonymous. Prodigal Prokaryotic Dynamic Programming Genefinding Algorithm. Oak Ridge National Laboratory and University of Tennessee 2009 http://compbio.ornl.gov/prodigal

23. Pati A, Ivanova N, Mikhailova, N, Ovchinikova G, Hooper SD, Lykidis A, Kyrpides NC. GenePRIMP: A Gene Prediction Improvement Pipeline for microbial genomes.. (Submitted)

24. Markowitz VM, Mavromatis K, Ivanova NN, Chen IM, Chu K, Kyrpides NC. IMG ER: a system for microbial genome annotation expert review ans curration. Bioinformatics 2009; 25: 2271-2278. PubMed PubMed doi:10.1093/bioinformatics/btp393 